\title{
Robotic single-site surgery versus laparoendoscopic single-site surgery in early-stage endometrial cancer: a case-control study
}

Hao Sun, Jinghai Gao, Zhijin Jin, Yuxian Wu, Yang Zhou, Xiaojun Liu

Department of Gynaecology and Obstetrics, Shanghai Changzheng Hospital, Shanghai, China

Videosurgery Miniinv 2021; 16 (3): 597-603

DOI: https://doi.org/10.5114/wiitm.2021.103955

\begin{abstract}
Introduction: Laparoendoscopic single-site surgery (LESS) can reduce the limited invasiveness of conventional laparoscopy while providing superior cosmetic results. Robotic single-site surgery (RSSS) can overcome this shortcoming to a certain extent.

Aim: To evaluate the advantages of RSSS in treating early-stage endometrial cancer by comparing RSSS with LESS. Material and methods: From January 2018 to August 2018, patients diagnosed with endometrial cancer from endometrial curettage and imaging studies were selected for this prospective cohort study, with 22 undergoing RSSS and 18 undergoing LESS. All surgical procedures were performed using the conventional da Vinci Si surgical system with the Lagiport single port or a conventional laparoendoscopic instrument with the Lagiport single port. Operative time was recorded electronically. Intraoperative parameters and postoperative parameters were recorded and further analyzed.

Results: The operation was successfully completed, and a pure single-point approach was adopted. There were no laparotomy or intraoperative complications. Compared with the LESS group, the RSSS group had significantly longer pre-surgical time, significantly lower median operation time, significantly lower median blood loss, and significantly lower vaginal cuff closure time. The median length of hospital stay in the RSSS group was significantly lower than that in the LESS group. There was no significant difference in the incidence of early and late complications between the two groups. No recurrence events were observed in either the RSSS or the LESS group.

Conclusions: RSSS is feasible and safe in patients with early-stage endometrial cancer. RSSS can reduce operating time, blood loss and length of hospital stay compared with LESS.
\end{abstract}

Key words: robotic, laparoendoscopic, single site, single port, endometrial cancer, gynecologic surgery.

\section{Introduction}

Recently, laparoscopy has moved forward with advancing technology. The first laparoscopic hysterectomy using a single incision was reported by Pelosi in 1991 [1]. It was not until general surgery began publishing success with appendectomies and cholecystectomies in the mid-2000s that laparoendoscopic single-site surgery (LESS) regained the application in gynecologic surgery [1]. Because the in- cision is hidden inside the umbilicus, it is considered as "scarless surgery". In addition, minimally invasive surgery improved post-operative pain and allowed for a prompt return to daily activities, thereby reducing hospitalization and overall medical expenses [2]. Casarin et al. presented the first case of minilaparoscopic single-site bilateral salpingo-oophorectomy (BSO) performed as a risk-reducing procedure [3]. However, despite being a more advanced surgical method, LESS still faces various surgical challenges,

\section{Address for correspondence}

Xiaojun Liu, Department of Gynaecology and Obstetrics, Shanghai Changzheng Hospital, Shanghai, China,

e-mail: liuxiaojun@smmu.edu.cn 
including a limited range of motion due to the parallel angle of the surgical instruments, and the difficulties in manipulating a flexible camera and surgical instruments in a limited space through a small skin incision [4-6]. In the past decade, robotic-assisted laparoscopic surgery has been accepted as an alternative method that can overcome the limitations of laparoscopic gynecologic surgery by providing improved precision, dexterity and a three dimensional view [7-9]. For obese patients with endometrial cancer, robotic surgery is feasible and safe [10]. However, there are few studies about the application of robotic single-site surgery (RSSS) in gynecologic oncology. Preliminary studies have proven the feasibility and safety of this approach for treating gynecological malignancies [11].

Endometrial cancer (EC) is one of the most common gynecologic malignancies. It is the third most common gynecologic cancer in Chinese women after carcinoma of the uterine cervix and ovarian cancer. The standard treatment for early-stage endometrial cancer is surgery, including laparotomy, laparoendoscopic surgery and robotic surgery. As mentioned previously, only a few reports about RSSS in gynecologic oncology have been published. Current trends in minimally invasive surgery are focused on decreasing surgical trauma through the elimination of incisions, thereby reducing postoperative discomfort, decreasing hospital stays, improving cosmetic results, and reducing wound-related complications $[12,13]$.

\section{Aim}

This study aimed to evaluate the advantages of RSSS in treating early-stage endometrial cancer by comparing RSSS with LESS. Additionally, our RSSS involved surgery with a combination of the conventional da Vinci Si surgical system and Lagiport single-port system, which achieved certain results and some surgical skills.

\section{Material and methods}

\section{Patients}

All patients enrolled in this retrospective study were scheduled to undergo RSSS or LESS in our hospital in the period from January 2018 to August 2018. All patients were informed about the RSSS or LESS techniques, benefits and related risks of possible laparoscopic or laparotomic conversion, and signed a written consent form. From January 2018 to August 2018, a total of 22 women who underwent RSSS were matched with 18 historic controls treated by LESS in the same institution, and these operations were performed by the same surgical team. All patients voluntarily chose surgical procedures after understanding the characteristics of each type of surgery.

Inclusion criteria were as follows: 1) no evidence of metastasis to other organs in the preoperative imaging; 2) a uterus size smaller than 12 gestational weeks; 3 ) endometrial cancer diagnosed by preoperative endometrial curettage or biopsy (International Federation of Gynecology and Obstetrics stages IA to IB) [14].

The standard exclusion criteria used for any laparoscopic cancer surgery were applied, but there were no restrictions related to body mass index (BMI) or previous abdominal surgery. Exclusion criteria were as follows: 1) supposed extensive adherences; 2) large uteri requiring morcellation; 3) very morbidly obese women who could not sustain a steep Trendelenburg position.

\section{Surgical technique}

\section{Surgical technique for RSSS}

Our team combined the conventional Da Vinci robotic system with the Lagiport single-port system to perform RSSS for the first time in China. The standard treatment for early-stage endometrial cancer is surgery, including a total hysterectomy, bilateral salpingo-oophorectomy, and pelvic and/or para-aortic lymph node dissection [13].

The first step started with an initial access; a $3 \mathrm{~cm}$ long incision was made over the lower rim of the umbilicus down to the level of the fascia, which was opened along the longitudinal axis of the body. The Lagiport was inserted into the incision and supported the space relying on its own elasticity. Next, the da Vinci Si surgical system was connected to the Lagiport. Pneumoperitoneum was established after insufflation of the abdomen up to a pressure of $15 \mathrm{~mm} \mathrm{Hg}$ and the table was placed in the Trendelenburg position $\left(30^{\circ}\right)$. A $12 \mathrm{~mm}$ endoscope cannula, a $8 \mathrm{~mm}$ bipolar grasper and $8 \mathrm{~mm}$ monopolar scissors were inserted vertically (Photos 1,2 ).

In the second step, the bilateral infundibulopelvic were skeletonized and transected $2 \mathrm{~cm}$ from the palace angle. The round ligaments were ligated 


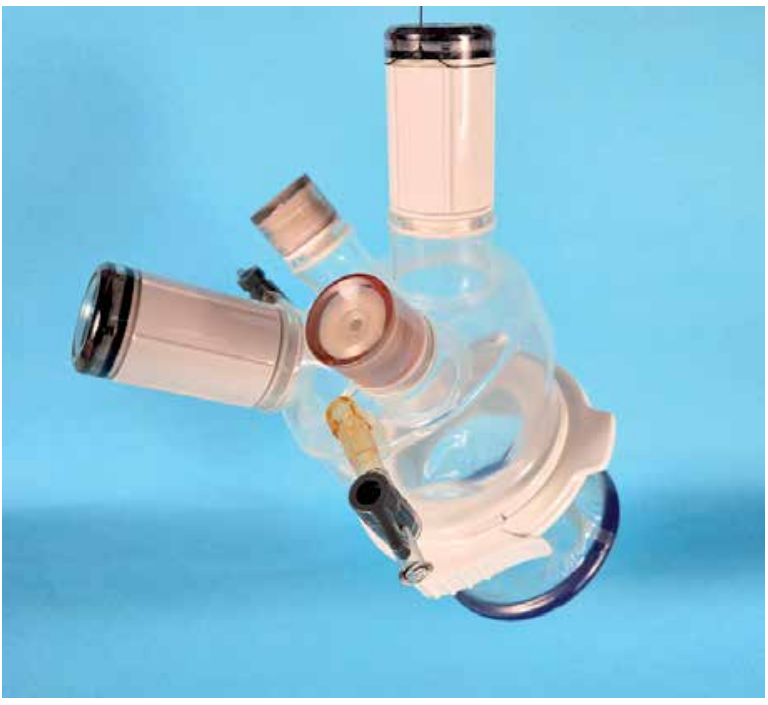

Photo 1. Lagiport single port

bilaterally. The bladder and the attached peritoneal flap were developed with the $8 \mathrm{~mm}$ monopolar scissors. Then, both uterine vessels were skeletonized and desiccated, including the uterine blood vessels and ligaments. Once the bladder had been dissected below the colpotomy cup, circumferential colpotomy was performed using the bipolar grasper and monopolar scissors. The uterus and the adnexa

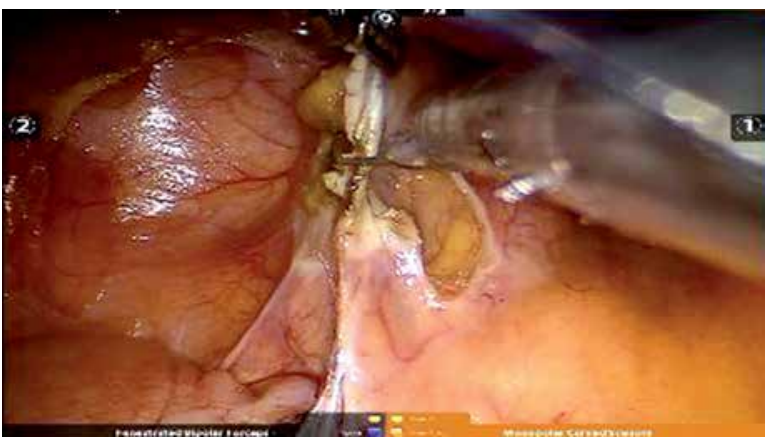

Photo 3. Ligation of infundibulopelvic ligament

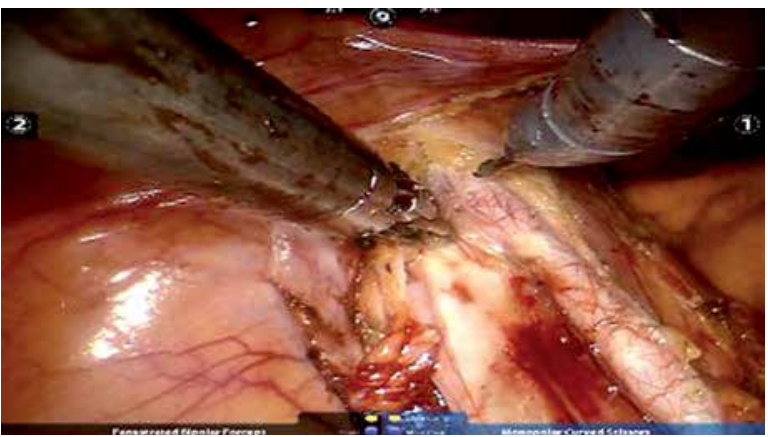

Photo 5. Pelvic/aortic lymphadenectomy

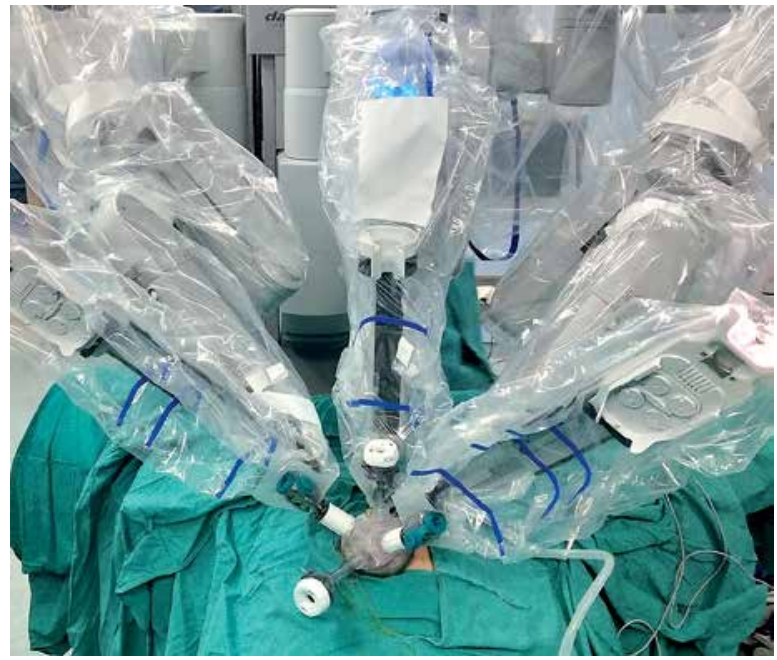

Photo 2. da Vinci Si surgical system with the Lagiport

were extracted through the vagina and sent for frozen section analysis (Photos 3, 4). For closure of the vaginal cuff, we applied continuous running suture intracorporeally using a barbed suture. After completion of the surgery, the fascial defect was closed with a delayed absorbable suture in a running or interrupted fashion. The skin was closed with an absorbable suture in a subcuticular or interrupted

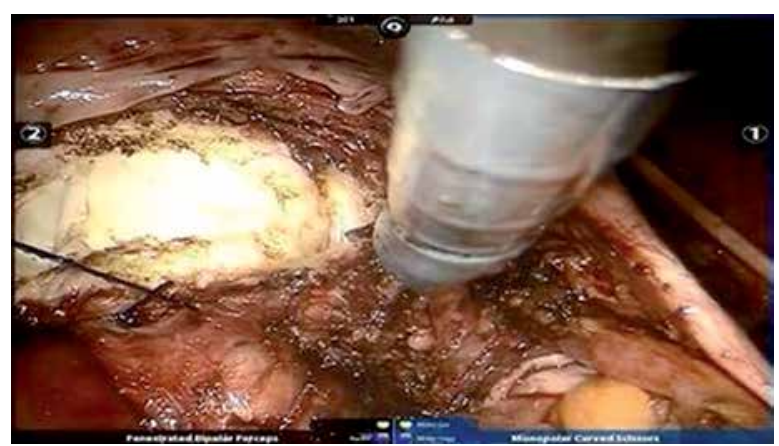

Photo 4. Circumferential colpotomy

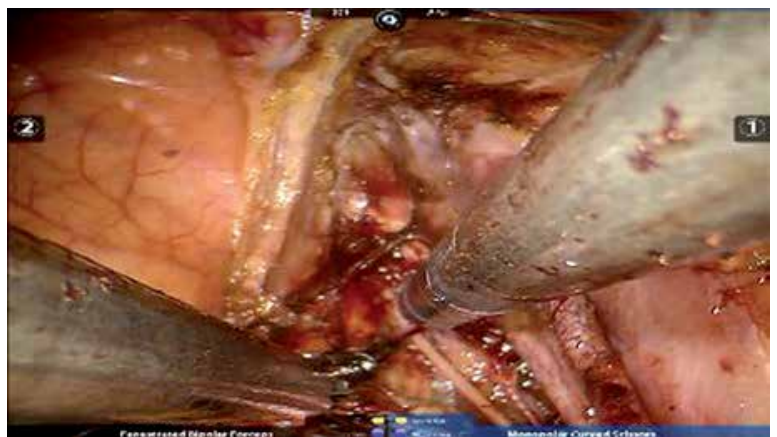

Photo 6. Exposure of obturator nerve 
fashion. A drainage tube was inserted through the vaginal stump. According to the literature data, we performed pelvic/aortic lymphadenectomy only in high-risk endometrial cancer [15] (Photos 5, 6).

\section{Surgical technique for LESS}

The team that performed the LESS was the same as the one that performed the RSSS. The first step started with an initial access, a $3 \mathrm{~cm}$ long incision was made over the lower rim of the umbilicus down to the level of the fascia, which was opened along the longitudinal axis of the body. The Lagiport was inserted into the incision and supported the space relying on its own elasticity. Pneumoperitoneum was established after insufflation of the abdomen to a pressure of $15 \mathrm{~mm} \mathrm{Hg}$ and the table was placed in the Trendelenburg position $\left(30^{\circ}\right)$. The $5-\mathrm{mm} 30^{\circ}$ telescope, grasper, cold scissors, suction and irrigation bipolar coagulator were inserted into the single port. The operating procedure was performed as described before.

\section{Perioperative and postoperative parameters}

Operative time, which was defined as the interval between the start of the incision and the closure, was recorded electronically. In addition, the time taken to perform umbilical incision and single-port placement and robotic docking, which we called

Table I. Clinical characteristics of patients

\begin{tabular}{|c|c|c|c|}
\hline Characteristics & $\operatorname{RSSS}(n=22)$ & $\operatorname{LESS}(n=18)$ & $P$-value \\
\hline Age [years]: & & & 0.908 \\
\hline Median & 56 & 55.5 & \\
\hline Range, IQR & $45-60,2$ & $43-62,5$ & \\
\hline BMI $\left[\mathrm{kg} / \mathrm{m}^{2}\right]$ : & & & 0.301 \\
\hline Median & 25.0 & 24.5 & \\
\hline Range, IQR & $19.0-31.0,4.0$ & 18.0-31.0, 5.8 & \\
\hline \multicolumn{2}{|c|}{ Previous abdominal surgery: } & & 0.919 \\
\hline Yes & 7 & 6 & \\
\hline No & 15 & 12 & \\
\hline FIGO stage: & & & 0.677 \\
\hline IA & 16 & 12 & \\
\hline IB & 6 & 6 & \\
\hline
\end{tabular}

$B M I$ - body mass index, FIGO - The International Federation of Gynecology and Obstetrics, IQR - interquartile range, RSSS - robotic single-site surgery, LESS - laparoendoscopic single-site surgery. pre-surgical time, was recorded. Console time was recorded by the circulating nurse, and was defined as the time during which robotic assistance was in use by the surgeon. The total operation time was calculated from setting time to console time.

Intraoperative parameters included estimated blood loss, total operative time and vaginal cuff closure time. Postoperative parameters included length of hospital stay, complications and median postoperative pain score.

In this study, operative time and estimated blood loss were observed as the primary outcome. Pre-surgical time, vaginal cuff closure time, length of hospital stay and median postoperative pain score were observed as the secondary outcomes. All patients had no conversion to multiport laparoscopy or laparotomy and were followed up at the outpatient clinic 2 weeks and 4 weeks after discharge.

\section{Ethics}

The study protocol was approved by the Ethics Committee of Changzheng Hospital, Second Military Medical University.

\section{Statistical analysis}

All the data collected were analyzed using SPSS 22.0 software. Measurement data were expressed as median and interquartile range (IQR) when appropriate (non-parametric distribution), and the comparisons were examined by Student's $t$ test and the Mann-Whitney test. The categorical data were expressed as $n$ (\%), and the differences between the two groups were examined by $\chi^{2}$ analysis or Fisher's exact test. $P<0.05$ was considered statistically significant.

\section{Results}

\section{Baseline data characteristics of patients}

No pre-operative statistically significant differences were observed ( $p>0.05)$, as shown in Table I. Age, BMI, with/without previous abdominal surgery and FIGO stage are not decisive factors of the way surgery is chosen.

\section{Comparisons of perioperative parameters between RSSS group and LESS group}

As shown in Table II, the median operative time of the RSSS group and LESS group were 95 and 
125 min respectively. Compared with the LESS group, the RSSS group had significantly longer pre-surgical time ( 8 min vs. 2 min, $p<0.05$ ), significantly lower median blood loss (50 $\mathrm{ml}$ vs. $85 \mathrm{ml}, p<0.05)$, and significantly lower vaginal cuff closure time $(21 \mathrm{~min}$ vs. $30 \mathrm{~min}, p<0.05)$. No intraoperative complications were observed in either the RSSS or the LESS group. No conversion to laparotomy or laparoscopy was necessary.

Final pathologic findings were similar between the two groups in terms of FIGO stage and grading. Definitive histologic results confirmed the frozen section examination: all of the specimens were diagnosed as endometrioid adenocarcinoma FIGO stage IA $(72.7 \%$ in RSSS group and $66.7 \%$ in LESS group) or FIGO stage IB (17.3\% in RSSS and $33.3 \%$ in LESS). The median length of hospital stay in the RSSS group was significantly lower than that in the LESS group ( 2 days vs. 3 days, $p<0.05$ ).

We evaluated the patients' postoperative pain 12,24 and $48 \mathrm{~h}$ after surgery. The data were significant at $24 \mathrm{~h}$ after surgery, as shown in Table III.

\section{Comparisons of postoperative parameters between RSSS group and LESS group}

There was no significant difference in the incidence of early and late complications between the two groups $(p>0.05)$. The median follow-up time was 16 months (range: 12-20 months) for the RSSS group and LESS group. There were 3 (13.6\%) patients with high-risk disease (stage IB; G3) who underwent further adjuvant radiotherapy in the RSSS group, and 2 (11.1\%) patients in the LESS group. No recurrence events were observed in either the RSSS or the LESS group.

\section{Discussion}

Four-trocar laparoscopic technique is the most common approach for simple extrafascial hysterectomy in early endometrial cancer [16]. LESS aims to minimize the skin incision to gain access to the abdominal or pelvic cavities to perform surgical procedures, which may translate into a benefit for patients in terms of port-related complications, recovery time, pain, and cosmesis. Research has shown that compared with multiport laparoscopic surgery, we can achieve the same outcome when we perform the LESS procedures without the need for placement of additional ports or conversion to laparoscopy [17].
Casarin et al. found that systematic lymph node dissection has important survival significance for patients, and the presence of lymph node metastasis was the main adverse prognostic factor [18]. Mean-

Table II. Comparisons of surgical outcomes between RSSS group and LESS group

\begin{tabular}{|c|c|c|c|}
\hline Parameters & $\operatorname{RSSS}(n=22)$ & LESS $(n=18)$ & $P$-value \\
\hline $\begin{array}{l}\text { Pre-surgical } \\
\text { time [min]: }\end{array}$ & & & $<0.001$ \\
\hline Median & 8 & 4 & \\
\hline Range, IQR & $6-15,3.5$ & $2-8,3.1$ & \\
\hline $\begin{array}{l}\text { Operating time } \\
\text { [min]: }\end{array}$ & & & 0.006 \\
\hline Median & 95 & 125 & \\
\hline Range, IQR & $85-150,38$ & $95-150,26$ & \\
\hline $\begin{array}{l}\text { Vaginal cuff } \\
\text { closure time } \\
\text { [min]: }\end{array}$ & & & $<0.001$ \\
\hline Median & 21 & 30 & \\
\hline Range, IQR & $15-30,8$ & $20-40,6$ & \\
\hline Blood loss [ml]: & & & $<0.001$ \\
\hline Median & 50 & 85 & \\
\hline Range, IQR & $30-100,25$ & $50-120,26$ & \\
\hline $\begin{array}{l}\text { Hospital stay } \\
\text { [days]: }\end{array}$ & & & 0.021 \\
\hline Median & 2 & 3 & \\
\hline Range, IQR & $1-4,2$ & $1-5,2$ & \\
\hline $\begin{array}{l}\text { Rate of compli- } \\
\text { cations(\%) }\end{array}$ & 0 & 0 & \\
\hline
\end{tabular}

$I Q R$ - interquartile range, RSSS - robotic single-site surgery, LESS - laparoendoscopic single-site surgery.

Table III. Postoperative pain scores of patients

\begin{tabular}{|lccc|}
\hline $\begin{array}{l}\text { Postoperative } \\
\text { pain scores }\end{array}$ & RSSS $(n=22)$ & LESS $(n=18)$ & $P$-value \\
\hline $\begin{array}{l}12 \mathrm{~h} \text { [median } \\
\text { (range, IQR)] }\end{array}$ & $3(2-4,2)$ & $4(2-4,1)$ & 0.089 \\
\hline $\begin{array}{l}24 \mathrm{~h}[\text { median } \\
\text { (range, IQR)] }\end{array}$ & $2(1-3,1)$ & $3(1-4,1)$ & 0.030 \\
\hline $\begin{array}{l}48 \mathrm{~h}[\text { median } \\
\text { (range, IQR)] }\end{array}$ & $1(1-2,1)$ & $1(1-2,0.72)$ & 0.722 \\
\hline $\begin{array}{l}\text { Frequency of } \\
\text { painkillers given } \\
\text { [median } \\
\text { (range, IQR)] }\end{array}$ & $0(0-1,0)$ & $0(0-1,0)$ & 0.482 \\
\hline
\end{tabular}

$I Q R$ - interquartile range, RSSS - robotic single-site surgery, LESS - laparoendoscopic single-site surgery. 
while, it was found that preoperative imaging techniques such as magnetic resonance imaging (MRI) can improve the accuracy of hysteroscopy biopsy [19]. However, some features of LESS face some significant challenges compared with the standard laparoscopy. One is the "sword fighting" among instruments as the instruments are inserted into the abdomen through a single incision. Recently, the da Vinci surgery technique was introduced to clinical practice to perform urological and gynecological surgery, with encouraging preliminary results [20, 21]. It gains people's attentions because of the speed of the learning curve and comfortable ergonomics for surgery and better outcomes for the patients. However, there are still some disadvantages for da Vinci to be extensively used, such as the costs and numbers of port sites.

To our knowledge, our team is the first gynecological minimally invasive surgery team to combine the conventional da Vinci robotic system with the Lagiport single port to perform gynecological surgery. The conventional da Vinci robotic system has its own advantages of the flexible robot arm compared with the single-site robot system. Our practice used the Lagiport single port to fuse the advantages of the conventional da Vinci robotic system.

Regarding the comparison between RSSS and LESS, this is the first case-control study in the literature on this issue. These two types of surgery were performed by the same surgical team, which made it comparable in terms of perioperative and postoperative parameters. Evidence across multiple disciplines suggests that RSSS is feasible, safe and leads to comparable outcomes compared with conventional laparoscopy or robotic surgery [22]. Even though the sample size in our study is small, our results are comparable to those reported for LESS or robotic surgery in terms of operative outcomes [23]. This novel approach in the field of minimally invasive gynecologic surgery may be accepted gradually.

LESS has the obvious advantages of improved cosmetic results and less postoperative pain, but the disadvantages of the annoying conflict between the instruments and the three dimensional view [24]. Conventional robotic single site operation has been proven to overcome these shortcomings [25]. We also found that the conventional robotic single-site systems did not have the flexible wrist motion capabilities similar to multi-port robotic systems. Our team obtained the maximum benefit through com- bining the conventional da Vinci robotic system with the Lagiport single port. When the surgical scope is large, RSSS cannot be adjusted like the conventional da Vinci robotic system. RSSS was also accompanied by certain obstacles, especially when we dealt with larger areas. It cannot be adjusted among larger areas like the conventional da Vinci robotic system. Therefore, we divided the entire surgical process into several parts and moved to another after completing one part with the help of an assistant [26].

\section{Conclusions}

RSSS is safe and feasible in the treatment of early-stage endometrial cancer. RSSS has obvious advantages in terms of perioperative parameters, such as blood loss and operative time. Our RSSS can also be mastered after 8-16 cases. Avoiding the annoying conflict between the instrument and the three dimensional view may be the real advantage for RSSS. The costs of robotic surgery may be the maximal obstructive factor for robotic surgery to be widely performed. The surgical skills need to be further explored through more cases. Further research about the comparisons between our RSSS and conventional RSSS should be performed in the future, and more advantages of our RSSS need to be proved. Robotic single-site laparoendoscopic surgery will become more and more widespread in gynecologic surgery with the development of science and technique.

\section{Acknowledgments}

Hao Sun and Jinghai Gao contributed equally to this work.

\section{Conflict of interest}

The authors declare no conflict of interest.

\section{References}

1. Jackson T, Einarsson J. Single-port gynecologic surgery. Rev Obstet Gynecol 2010; 3: 133-9.

2. Laganà AS, Garzon S, D'Alterio MN, et al. Mini-laparoscopy or single-site robotic surgery in gynecology? Let's think out of the box. J Invest Surg 2020; 11: 1-2.

3. Casarin J, Laganà AS, Pinelli C, et al. Minilaparoscopic single-site bilateral salpingo-oophorectomy: a scarless prophylactic procedure. Minim Invasive Ther Allied Technol 2020; 116: 1-6.

4. Fader AN, Escobar PF. Laparoendoscopic single-site surgery (LESS) in gynecologic oncology: technique and initial report. Gynecol Oncol 2009; 114: 157-61. 
5. Fader AN, Cohen S, Escobar PF, et al. Laparoendoscopic single-site surgery in gynecology. Curr Opin Obstet Gynecol 2010; 22: 331-8.

6. Uppal S, Frumovitz M, Escobar P, et al. Laparoendoscopic single-site surgery in gynecology: review of literature and available technology. J Minim Invasive Gynecol 2011; 18: 12-23.

7. Advincula AP, Song A, Burke W, et al. Preliminary experience with robot-assisted laparoscopic myomectomy. J Am Assoc Gynecol Laparosc 2004; 11: 511-8.

8. Bedient CE, Magrina JF, Noble BN, et al. Comparison of robot ic and laparoscopic myomectomy. Am J Obstet Gynecol 2009; 201: 566.e1-5.

9. Nezhat C, Lavie O, Hsu S, et al. Robotic-assisted laparoscopic myomectomy compared with standard laparoscopic myomectomy - a retrospective matched control study. Fertil Steril 2009; 91: 556-9.

10. Zhao Y, Liu Z, Yu L, et al. Robotic surgery in obese patients with early-stage endometrial cancer. Videosurgery Miniinv 2020; 15: 171-5.

11. Lambaudie E, Cannone F, Bannier M, et al. Laparoscopic extraperitoneal aortic dissection: does single-port surgery offer the same possibilities as conventional laparoscopy? Surg Endosc 2012; 26: 1920-3.

12. Gettman MT, White WM, Aron M, et al. Where do we really stand with LESS and NOTES? Eur Urol 2011; 59: 231-4.

13. Wright JD, Barrena Medel NI, Sehouli J, et al. Contemporary management of endometrial cancer. Lancet 2012; 379: 1352-60.

14. Amant F, Mirza MR, Koskas M, et al. Cancer of the corpus uteri. Int J Gunaecol Obstet 2018; 143 (Suppl 2): 37-50.

15. Wright JD, Barrena Medel NI, Sehouli J, et al. Contemporary management of endometrial cancer. Lancet 2012; 379: 1352-60,

16. Chang WC, Lee LC, Huang SC, et al. Application of laroscopic surgery in gynecological oncology. J Forms Med Assoc 2010; 109: 558-66.

17. Fagotti A, Boruta II DM, Scambia G, et al. First 100 early endometrial cancer cases treated with laparoendoscopic single-site surgery: a multicentric retrospective study. Am J Obstet Gynecol 2012; 206: 353.e1-6.

18. Casarin J, Bogani G, Piovano E, et al. Survival implication of lymphadenectomy in patients surgically treated for apparent early-stage uterine serous carcinoma. J Gynecol Oncol 2020; 31: e64.

19. Cignini P, Vitale SG, Laganà AS, et al. Preoperative work-up for definition of lymph node risk involvement in early stage endometrial cancer: 5-year follow-up. Updates Surg 2017; 69: 75-82.

20. Konstantinidis KM, Hirides P, Hirides S, et al. Cholecystectomy using a novel single-site robotic platform: early experience from 45 consecutive cases. Surg Endosc 2012; 26: 2687-94.

21. White MA, Autorino R, Spana G, et al. Robotic laparoendoscopic single site urological surgery: analysis of 50 consecutive cases. J Urol 2012; 187: 1696-701.

22. Chung H, Jang TK, Nam SH, et al. Robotic single-site staging operation for early-stage endometrial cancer: initial experience at a single institution. Obstet Gynecol Sci 2019; 62: 149-56.

23. Fagotti A, Gagliardi ML, Fanfani F, et al. Perioperative outcomes of total laparoendoscopic single-site hysterectomy versus total robotic hysterectomy in endometrial cancer patients: a multicentre study. Gynecol Oncol 2012; 125: 552-5.

24. Jayakumaran J, Wiercinski K, Buffington C, et al. A. Robotic laparoendoscopic single-site benign gynecologic surgery: a single-center experience. J Robotic Surg 2017; 12: 447-54.

25. Fagotti A, Corrado G, Fanfani F, et al. Robotic single-site hysterectomy (RSS-H) vs. laparoendoscopic single-site hysterectomy (LESS-H) in early endometrial cancer: adouble-institution case-control study. Gynecol Oncol 2013; 130: 219-23.

26. Moukarzel LA, Fader AN, Tanner EJ. Feasibility of robotic-assisted laparoendoscopic single-site surgery in the gynecologic oncology setting. J Minim Invas Gyn 2017; 24: 258-63.

Received: 9.12.2020, accepted: 22.12.2020. 